\title{
DEUX TENTATIVES DE RECUPERATION DE LA "PENSEE-MICHEL-FOUCAULT"
}

Je voudrais donner les deux textes qui suivent pour des documents datés (1977). Ils traduisent, il me semble, jusque parfois dans leur vocabulaire et leur style, un moment particulierde la réception de l'oeuvre de Foucault ou plutôt de ce que l'on voulait alors appeler la "pensée-Michel-Foucault" (sur le modele de la "pensée-Mao-Tsé-toung"), le sujet s'effaçant derrière le monde ouvert par un "penser"qui était ressenti comme un travail collectif quasi anonyme. Je m'efforçais de "défendre" Foucault contre des déviances qui me paraissaient dommageables pour l'intégritéde son entreprise sans me rendre compte que je partageais au moins avec les "récupérateurs 'la conception d'une "pensée-Michel-Foucault" suffisamment transhistorique pour transcender son temps et son auteur et pour nous apporterl'illumination décisive. Aujourd'hui ma vision seraitplus critique et plus historique. J'éclaireraicertains points en étoffant un peu les notes originelles et en citant quelques passages des lettres que Foucault m'avait adressées à l'époque.

\section{MAITRE, POUVOIRS, DISCOURS, SEXUALITE}

(A propos de Christian Jambet: Apologie de Platon. Paris: Grasset, 1976 et de Michel Foucault: La volonté de savoir. Paris: Gallimard, 1976).

Un texte m'émeut, c'est-à-dire me met en mouvement:

J'imaginais d'écrire sur Foucault il y a trois ans, je fis de cette oeuvre l'occasion de poser de façon différente la question de la rébellion, de son histoire. ... Les livres de Foucault, chacun les prendra comme il voudra, selon l'humeur: bréviaires de la méthode, encouragements et plaisir à l'histoire, eléments d'une mémoire populaire, révolution de notre sentiment... Ils furent pour moi, avant tout cela, des paroles savantes, des paroles d'écrivain que je pouvais sans horreur morale accepter d'entendre: déshabitué de longtemps par l'expérience maoiste de lire et d'écrire, je ne trouvais pas, abandonné par elle, d'autres livres pour ne pas m'écoeurer. ${ }^{\prime}$

C'est là le premier moment d'une tentative d'annexion. Ensuite Christian Jambet évoque la période de "décervelage" maoiste et son retour à la

\footnotetext{
'Apologie de Platon, p. 37.
} 
pensée. Mais la péroraison du passage inquiète plus:

Michel Foucault éducateur: déracinée, chassée de l'histoire multiple du maître, l'Idée de révolte, que rien n'annonce, mais qui doit au contraire egendrer sa propre dimension, s'oppose aux positivités comme l'événement s'oppose au temps. Et non comme l'avènement, la réussite de leur signification enfouie. S'éduquer: prévenir toute confusion de la rébellion et du monde, de l'Ange et du Maître. C'est opposer une illumination à une lumière, une métaphysique négative à une mathésis, une ontologie du Deux à une politique du Un. ${ }^{2}$

Et voilà, sans plus de façons, Michel Foucault embrigadé dans les cohortes de $1^{\text {' Ange": }}{ }^{3}$ du moins la présentation ambiguë de cette "filiation spirituelle" le laisse-t-elle supposer. Il y a là, me semble-t-il, un grave contresens sur la portée de l'oeuvre et de la pensée de Michel Foucault, tout comme sur leur "bon usage". Son tout dernier livre, premier tome d'une impressionnante entreprise, porte très clairement le coup de grâce à tout détournement de ce genre. Mais Christian Jambet n'en est pas en la matière à son premier essai, ${ }^{4}$ ni au dernier: ne nous annonce-t-on pas un Michel Foucault éducateur!

\section{PLATON ENTRE L'ANGEET LE GOULAG}

C'est principalement Platon--puisque le livre de Jambet lui doit son titre - qui subit les effets de l'étonnante hardiesse synthétique de l'essayiste. L'on part d'une remouture a peine nuancée du Platon scolaire, divisé pour la forme en deux moments théoriques que l'on oppose: la période de la "Théorie des Idées" qui trouve son sommet dans la

\footnotetext{
${ }^{2}$ Ibid., p. 40.
}

3 (3) La nature de l' "Ange"est à peine définie dans le livre de Guy Lardreau et Christian Jambet qui porte ce titre (Paris, Grasset: 1976). Ce que l'on en dit de plus précis est ceci: "...contre toutes les puissances et les dominations, maintenir l'espoir qu'un autre monde, malgré tout, est possible. A désigner cette possibilité, nulle autre image ne s'imposa à nous que celle de l'"Ange" $(p, 10)$. L'Ange est donc le symbole "incarné" de la rébellion contre tout pouvoir, contre toute immanence--d'une rébellion qui en appelle à la transcendance du possible sur le réel. Nos deux auteurs ont, depuis choisi la voie de la mystique orientale telle qu'Henry Corbin l'a dévoilée aux yeux de l'Occident: leur vision de la transcendance a revétu les formes et les voies de l'angélologie iranienne (d'origine zoroastrienne).

4(4) Voir, à ce propos, la vigoureuse réplique de Laurent Dispot aux arguments de L'Ange: "La Bête," Critique, nº 355, décembre 1976. 
dialectique ascendante mise en oeuvre dans la République et une période où le beau schéma se trouble au profit d'une théorie médiane parce que l'inscription des Idées-Nombres au Ciel des Fixes rend le décalage si net entre toute Idée et sa réalisation matérielle qu'aucune dialectique ne peut plus permettre l'accession à l'Idée-même--période du Timée et des Lois que l'on fait commencer avec la réfutation des thèses éléatiques du Parménide. ${ }^{5}$ Le point de renversement réside dans le changement qui affecte l'Idée du Bien: elle n'est plus ce soleil de l'Etre que l'évadé de la caverne pouvait encore presque soutenir du regard mais l'abstraction pure et simple de l'Un-Bien-Beau, à jamais inaccessible et dont on ne saurait tirer que des copies et des copies de copies de plus en plus falotes, par l'entremise d'un Démiurge pétrisseur de formes mais qui n'a pas créé les Formes premières. A partir de ce revirement, Christian Jambet trace les voies de deux platonismes diamétralement opposés: le premier serait le précurseur illuminant des dialectiques négatives propres à certaines pensées dualistes, le second au contraire ferait le lit de l'aristotélisme (ici chargé de tout le mal) qui se réduit pour Jambet à une prudente, trop prudente, gestion du possible. La démonstration se veut claire, pour ne pas dire simpliste, elle ne vise qu'à étayer une manière d'a priori théorique: il y a un Platon de l' "Ange",du "Rebelle", de l' "Un"par excès (celui qui dans le secret de son coeur soutient tout de même la première hypothèse du Parménide) et un Platon du "Multiple",du "Maître",gauchi, voué à un type de "récupération" symbolisé par Aristote, un Platon qui serait l'instituteur de tous les Goulags du monde dont on pourrait d'ailleurs entrevoir la logique et la structure dans certains préceptes de gouvernement propres aux Lois (et peut-être déjà à la République!). Certes il n'est pas difficile de lire rétroactivement la dialectique platonicienne à partir de la procession plotinienne, mais à quoi sert ce Platon nouveau, fabriqué pour les besoins de la cause "angélique"? Pour l'"angéliste" convaincu, il n'est ici que le prétexte d'un exercice de vocalises, d'une litanie déroulant de beaux et savants répons--glissandi théoriques qui permettent au "nouveau gourou" de titiller Leibniz, Locke et Rousseau, voire Bergson, pour révéler par un ultime tour de passe-passe que tout ça n'est autre qu'une répétition générale de la G.R.C.P. (Grande Révolution Culturelle Prolétarienne). Curieuse façon de prendre l'histoire à rebrousse-poil: comment le même auteur peut-il vanter, et du même élan, les "encouragements et [le] plaisir à l'histoire" qu'il décèle chez

'(5) A propos de l'Un chez Platon et de l'actualité de Parménide-- du Parménide--voir Charles Singevin, Essai sur l'Un. Paris: Seuil, 1969. 
Michel Foucault? On peut se demander ce qui dans l'oeuvre de ce dernier induit pareille dérive.

\title{
"LAPENSEE DU DEHORS"
}

Le seul point par où Michel Foucault pourrait peut-être se rapprocher de la conception de 1' "Ange" propre à Lardreau et Jambet, serait cette "pensée du dehors" analysée dans un texte consacré à 1'oeuvre de Maurice Blanchot. ${ }^{6}$ Dans ce bel essai, Michel Foucault décrit de la façon la plus nette le statut périlleux de celui qui, à notre époque, prend le risque de dire ou d'écrire "Je parle." En cette seule énonciation se dévoile une telle expérience du langage--elle appartient à notre épistémé, à notre configuration de savoir--que qui écrit ou dit "Je"se trouve aussitôt confronté à une parole qui l'excède largement et vite l'exténue: "l'être du langage n'apparait pour lui-même que dans la disparition du sujet. ${ }^{77}$ Soutenir ce rapport au langage en son être pourrait proprement s'appeler une "pensée du dehors." Michel Foucault émet alors l'hypothèse que ce type de pensée a sans doute un répondant millénaire et quasi mystique:

\begin{abstract}
On peut bien supposer qu'elle est née de cette pensée mystique qui depuis les textes du Pseudo-Denys, a rôdé aux confins du christianisme; peut-être s'est-elle maintenue, pendant un millénaire ou presque, sous les formes d'une théologie négative. Encore n'y a-t-il rien de moins sur: car si dans une telle expérience il s'agit bien de passer "hors de soi, "c'est pour se retrouver finalement, s'envelopper et se recueillir dans l'intériorité éblouissante d'une pensée qui est de plein droit Etre et Parole, Discours donc, même si elle est, au-delà de tout langage, silence, au-delà de tout être, néant. ${ }^{8}$
\end{abstract}

Pressentant le risque que serait l'assimilation trop rapide et abusive de ce mode de pensée (de ce que Blanchot appelle "la pensée du neutre") à la voie négative des mystiques, Michel Foucault rétablit tout de suite les distances. C'est là, très précisément, esquiver le piège où sombre la tentative d' "élévation"de Christian Jambet. Son idéal "angélique" qui se voudrait proche dans les termes et dans la pratique d'une telle "pensée du dehors," revêt trop servilement l'oripeau mystique d'un dualisme

${ }^{6}$ (6) "La Pensée du dehors, " Critique, No. 229, iuin 1966, pp, 523-546.

${ }^{7}$ p, 525.

${ }^{8}$ p. 526. 
manichéen, de forme cathare, et se laisse emporter par l'illusion d'une rémunération intime, d'une sorte de rédemption immmédiate, bien que masquée. C'est ce que nous donne à lire "l'Histoire de Théophile et de Marie, "placée en appendice, en guise de stade premier pour l' "élévation" souhaitée. Certes, les deux personnages errants que dépeint cette histoire édifiante sont pauvres, chastes et ils prennent bien soin de se faire quotidiennement conspuer par la populace des villes qu'ils traversent (en habits de saltimbanques, avec une attitude dépravée et provocante): ils vivent une extrême humiliation qui socialement et individuellement les réduit à néant. Pourtant ce fatras et cette comédie ne sont-ils pas le meilleur rempart que nos deux mystiques aient pu trouver pour préserver leur exquise intériorité, leur vérité profonde et ineffable, enclose en leur coeur qu'elle illumine. Fausse quête donc de celui qui a déjà en lui sa lumière et qui fait tout pour la garder à soi, pour soi. Egoïsme mystique d'une sorte de "riche en esprit" qui dans son for intérieur se moque tant des benêts qui le couvrent de boue et de crachats-l'assurance de qui sait déjà, en face de ceux qui continuent à errer et qu'il y a plaisir à laisser ou même à faire errer.

Cette mise à nu rend aux prétentions mystiques de Jambet leur dimension véritable: la fausse attente d'êtres trop sûrs d'eux et de leur vérité pour n'être pas dans le fond dominateurs, même si cette domination s'établit surtout négativement, par l'absence d'intervention ou la volonté de silence. Résurrection, par l'entremise de vieux mythes rémunérateurs, d'une consolation morale, d'un viatique pour nos temps difficiles, mais avec la certitude intime de n'être pas les barbares, de n'être pas du côté du Maître, certitude qui vaut bien celle du Maître, un tranquille Pereant Barbari dont on s'exclut soi et les siens. ${ }^{9}$

\section{FOUCAULTLE DERACINEUR}

Contre une telle propension à la dictature camouflée sous une apparente humilité, comment situer 1'entreprise théorique de Michel Foucault? Tout d'abord, malgré d'indéniables réussites, elle reste humble et honnête, ne prétendant jamais à un savoir illuminateur ou arrogant, fidèle en cela à la "pensée du dehors" qui est sa limite. Elle garde l'allure d'une tâche quotidienne menée avec le constant souci de comprendre, de

\footnotetext{
${ }^{9}$ De nos jours (1993), nous lisons avec plus d'acuité le lien entre une telle certitude intérieure qui s'accompagne d'un violent mépris pour l'ignorant ou le pécheur, pour l'autre que soi, et le fanatisme propre à tous les intégrismes déchaînés: pour passer d'une attitude à l'autre il suffit en fait d'extérioriser sa certitude et de s'en faire une arme.
} 
débroussailler régionalement l'enchevêtrement des problèmes qu'elle se pose. La "boîte à outils," dont chacun peut utiliser à son gré les instruments divers, reste la meilleure image qui puisse rendre compte de la finalité de l'entreprise.

Son domaine est le massif ensemble des discours et leur entassement sous forme d'archives: tout ce que les siècles de notre longue histoire nous ont légué en fait de traces écrites. A charge pour le "nouvel archiviste" de découper son objet dans cet amas mal différencié, choix délicat qui opère plus selon de grands partages lisibles en filigrane--les épistémès--que selon les scansions traditionnelles d'une science historique solidifiée en disciplines et en périodes. Mais l'hypothèse qui inspire et informe tout ce labeur patient et minutieux est le pari fait sur un ordre du discours qui tend à faire reconnaître, d'une part, ce qui appartient au discursif comme tel et, d'autre part, un certain type de régulation et de circulation propre à ce discursif:

\begin{abstract}
...je suppose que dans toute société la production du discours est à la fois contrôlée, sélectionnée, organisée et redistribuée par un certain nombre de procédures qui ont pour rôle d'en conjurer les pouvoirs et les dangers, d'en maítriser l'événenent aléatoire, d'en esquiver la lourde, la redoutable matérialité. ${ }^{10}$
\end{abstract}

Le tissu serré du discours et des institutions, du réel et des mots, leur enchevêtrement complexe, surtout dans les domaines où le discours "mord" tout de suite sur le réel sous forme de codes, de règlements, de manuels, de jugements pénaux, est mis au jour, en transparence. Il s'en dégage une conception nouvelle du pouvoir tant dans son rapport au savoir que dans son rapport à ses objets propres. Les décrochages et réajustements locaux entre les séries discursives et les institutions sociales offrent d'infinies modulations possibles, et il faut désormais écrire pouvoirs, ou même micropouvoirs, à audience locale--raffinement de l'analyse qui décentre le problème de la maîtrise pour le déchiffrer dans l'équilibre (ou le déséquilibre ou le statu quo) propre à la vie quotidienne, dans le jeu de bascule perpétuel entre discours, techniques des pouvoirs et résistances:

Faut-il dire ...que, l'histoire étant la ruse de la raison, le pouvoir, lui, serait la ruse de l'histoire--celui qui toujours gagne? Ce serait méconnaître le caractère strictement relationnel des rapports de pouvoir. Ils ne peuvent exister qu'en fonction d'une multiplicité de

\footnotetext{
${ }^{10}$ Michel Foucault, L'ordre du discours, Paris: Gallimard, 1971,p, 1.
} 
points de résistance: ceux-ci jouent dans les relations de pouvoir, le rôle d'adversaire, de cible, d'appui, de saillie pour une prise. Ces points de résistance sont présents partout dans le réseau du pouvoir. Il n'y a donc pas par rapport au pouvoir un lieu du grand Refus--âme de la révolte, foyer de toutes les rébellions, loi pure du révolutionnaire. Mais des résistances qui sont des cas d'espèces: possibles, nécessaires, improbables, spontanées, sauvages, solitaires, concertées, rampantes, violentes, irréconciliables, promptes à la transaction, intéressees, ou sacrificielles; par définition, elles ne peuvent exister que dans le champ stratégique des relations de pouvoir. Mais cela ne veut pas dire qu'elles n'en sont que le contrecoup, la marque en creux, formant par rapport à l'essentielle domination un envers finalement toujours passif, voué a l'indéfinie défaite. Les résistances ne relèvent pas de quelques principes hétérogènes; mais elles ne sont pas pour autant leurre ou promesse nécessairement déçue. Elles sont l'autre terme, dans les relations de pouvoir; elles s'y inscrivent comme l'irréductible vis-à-vis."

Cette analyse qui démultiplie les lieux, les moments et les moyens des pouvoirs en même temps que leurs points vifs d'articulation et de résistance évacue comme caduque la figure maudite du Maitre invétéré, rend puéril le partage du monde en deux camps affrontés de toute éternité, camp des riches et camp des pauvres, des forts et des faibles, du capital et des prolétaires, des élus et des infidèles. La conception toute juridique du pouvoir qui sous-tend une telle vision dualiste est remise en question. Le pouvoir n'est plus conçu sur le modèle de la loi; il n'a plus rien de la machine binaire à interdire ou à tolérer: il est le produit de relations indissolubles et multiples que font sans cesse évoluer les remaniements tactiques des protagonistes. Il ne pourrait y avoir de "Maitre" et d' "Ange" que dans une situation de terreur continuelle où l'un excluant absolument l'autre, se livrerait une lutte à mort qui verrait vite l'épuisement des deux antagonistes.

Il est plus difficile sans doute--car c'est un effort de chaque jour-d'envisager, c'est-à-dire de tenir sous son regard, la réalité complexe des forces en présence ou beaucoup se règle au coup par coup, au niveau local, dans des configurations de savoir et de pouvoir toujours singulières. C'est en cela que Michel Foucault peut être dit déracineur: il met au jour l'entrecroisement constant des discours et des pouvoirs aux divers moments de l'histoire, et surtout, arrachant ces derniers à leurs sites traditionnels (discipline, chronologie, ordre des raisons, partage entre particulier et général...), il introduit un décalage tel qu'il fait flageoler tous

\footnotetext{
"La volonté de savoir, p. 126-127.
} 
les objets conventionnellement découpés et vaciller les socles qui les portaient: il met en perspective. En particulier la thèse qu'il développe dans son dernier livre La volonté de savoir, à propos du discours sexuel occidental, veut provoquer et bousculer avec allégresse ceux qui se sont si bien installés aux lieu et place des Pères.

\section{FREUD MIS EN PERSPECTIVE}

La sexualité (ce que nous appelons ainsi) a été proprement inventée: elle n'est pas un fait de nature mais le produit d'un réseau culturel et historique dont Michel Foucault se proposait de dénouer les mailles dans les six tomes annoncés au moment où il publiait le premier volume de son Histoire de la sexualité. Elle a été inventée par l'Occident chrétien à travers, en particulier, l'exigence instante de l'aveu, de la confession plus ou moins extorquée au fidèle, recherche interminable des racines de la primitive concupiscence, celle de la chair évidemment, au principe même de tout péché. En repérant point par point le cheminement historique de cette sexualisation de l'être humain, Michel Foucault développe une critique rigoureuse de la conception courante qui proclame que le sexe a toujours été réprimé. Pour lui, il n'y pas eu répression au sens propre du terme puisqu'il n'existe pas de sexualité naturelle, à l'état sauvage: il y a eu, au contraire, mise en place d'un dispositif complexe d'assujettissement, de rabattement de tout ce qui concerne le corps et la vie de l'âme sur le sexe, en même temps que s'instaurait un modèle normatif de régulation des fonctions sexuelles. Le projet de Michel Foucault est d'étudier dans tout son détail la montée au cours des trois derniers siècles d'un discours du sexe (loin de toute répression au sens propre, l'on parle en effet et l'on fait de plus en plus parler le sexe). Emergence d'une volonté de savoir qui se tourne vers le sexe comme vers le lieu du secret et produit en même temps l'idéologie du sexe opprimé. En fait, le but des pouvoirs est de saine gestion du cheptel social à travers une socialisation toujours accrue du sexuel. C'est de ce discours-là, propre à l'Occident chrétien que nait le concept de sexualité: on peut supposer à juste titre que d'autres civilisations, comme la civilisation arabomusulmane par exemple, n'ont pas au sens strict de sexualité, mais un quadrillage sexuel autre qui n'a pas développé les 
mêmes entrecroisements de discours, d'institutions et de pratiques réelles. ${ }^{12}$

Le but d'un tel déracinement théorique est de mettre en évidence, à travers les discours du sexe propres à l'Occident, la grande continuité d'une telle quête du secret, et de rendre à leur perspective véritable certains des monstres sacrés, des révolutionnaires prétendus de cette quête:

\begin{abstract}
On se moquera du reproche de pansexualisme qui fut un temps objecté à Freud et à la psychanalyse. Mais ceux qui paraittront aveugles seront peut-être moins ceux qui l'ont formulé que ceux qui l'ont écarté d'un revers de main, comme s'il traduisait seulement les frayeurs d'une vieille pudibonderie. Car les premiers, après tout, ont été seulement surpris par un processus qui avait commencé depuis bien longtemps et dont ils n'avaient pas vu qu'il les entourait déja de toutes parts; ils avaient attribué au seul mauvais génie de Freud ce qui avait été préparé de longue main; ils s'étaient trompés de date quant à la mise en place, dans notre société, d'un dispositif général de sexualité. Mais les seconds, eux, ont fait erreur sur la nature du processus; ils ont cru que Freud restituait enfin au sexe, par un retournement soudain, la part qui lui était due et qui lui avait si longtemps été contestée; ils n'ont pas vu que le bon génie de Freud l'avait placé en un des points décisifs marqués depuis le XVIIIème siècle par les stratégies de savoir et de pouvoir; et qu'il relançait ainsi avec une efficacité admirable, digne des plus grands spirituels et directeurs de l'époque classique. l'injonction séculaire d'avoir à connaître le sexe et à le mettre en discours. ${ }^{13}$
\end{abstract}

On aurait pu pousser plus loin et, dans notre modernité, remettre Lacan, lui aussi, dans la perspective; ce qui aurait ôté bien vite aux "angélistes" l'un des garants qu'ils aimaient le plus à solliciter. C'est dire que nous attendions avec impatience les autres volumes de l'Histoire de la sexualité!

\title{
MICHEL FOUCAULT EDUCATEUR
}

Toute l'importance de l'ébranlement provoqué ici par Michel Foucault n'est pas encore discernable. L'effet produit sur le lecteur de bonne foi est d'abord de vertige, est dans un mouvement subit,toute une histoire qui bascule et reprend forme sous nos yeux, remodelée.

12 Voir A. Buhdiba: La sexualité en islam, Paris, PUF, 1975, et notre compte-rendu "Société musulmane et quadrillage sexuel, " Critique, No. 349-35, juin-juillet 1976.

${ }^{13}$ La volonté de savoir, pp. 209-210. 
Ebranlement, étonnement, dépaysement culturel, déracinement intellectuel: tels sont les événements éminemment pédagogiques, formateurs que suscite cette lecture.

Nous sommes quelques-uns que le choc dû à l'étude de ces deux livres complémentaires que sont Les mots et les choses et $L$ 'archéologiedu savoir a arraché, non à un lourd sommeil dogmatique, mais à leurs songes juvéniles. Nous y avons appris--outre tout ce que l'on peut y apprendre sur notre histoire! --à ne plus avoir honte d'être des "intellectuels." ${ }^{14}$ A accepter d'être à notre tour "gens de l'archive," que ce soit comme "archivistes" ou comme producteurs de discours. A ne pas avoir peur de n'être en tout et pour tout que des "effets" de parole ou d'écriture parmi d'autres traces, d'autres échos. A nous insérer dans la trame ou dans le tissu d'un discours sans commencement ni fin qui nous cerne et nous emporte.

La révélation que le champ du politique tout comme celui de l'histoire sont en tous points tissés de discursif et de social, de mots et de choses, de relatif et de relationnel, nous a donné un sens plus juste du possible et du souhaitable. Nous insérer comme point de résistance actif dans le jeu parfois ténu des micropouvoirs, avec autant d'humilité qu'il y avait d'arrogance dans les totalisations forcenées auxquelles nous voulûmes d'abord croire, et provoquer à notre propre niveau d'intervention, fût-ce dans un contexte microscopique, un ébranlement analogue à celui que crée Michel Foucault dans les plus hautes instances du culturel, pour nous ce serait déja beaucoup.

Nous ne pouvons donc ici que nous démarquer vigoureusement-au nom de notre histoire et d'une certaine idée de l'histoire que nous devons à Foucault--des tentatives de récupération "angélistes" qui tendent et tendront encore a défigurer la portée de son entreprise.

(janvier 1977)

Dans mon esprit, ce papier était destiné à Critique qui venait de publiermon artitlesur Société musulmane et quadrillage sexuel (juin-juillet 1976). Mais

\footnotetext{
" Dépassant la définition de l' "intellectuel" (héritée du XIXème siècle et de l'affaire Dreyfus) qui le veut guide éclairé des masses en raison de son éminent rapport à la vérité (qui devient ainsi un pouvoir), Foucault propose une conception de l'"intellectuel spécifique" qui intervient comme point de résistance dans le jeu complexe des micropouvoirs, moins en raison de son rapport au vrai et au bien que de son insertion "spécifique" dans la pratique "technologique"--le logos pratique--des forces et des formes sociales.
} 
une brouille étant intervenue entre Jean Piel, directeur de Critique et Foucault, ce ne fut pas possible. Mon texte plut toutefois assez a Foucault pour qu'il ait envie de me renrontrer. Notre entrevue eut lieu d la mi-février 1977. Cette eonversation me fit prendre conscience des enjeux politiques très concrets liés à l'entreprise et à la situation du philosophe et l'intellectuel Foucault à ce moment précis. L'émergence toute récente des "nouveaux philosophes, "auxquels d'une certaine façon, Lardreau et Jambet s'étaient ralliés (au moins éditorialement), était considérée par ses amis de gauche comme une tentative idéologique, appuyée sur les média, pour discréditerla toute récente "union de la gauche. "Il était de la meilleure stratégied'essayer d'embrigader "Foucault d'un côté ou de l'autre. Ce dernier me sembla fort agacé d'être ainsi pris en otage et il était irrité par de continuelles sollicitations:pétitions à signer, interventions dans des manifestations et des réunions politiques, des débats, des grèves...ll souhaitait très clairement se démarquer des "nouveaux philosophes" qui déformaient sa pensée mais sa conception de l'engagementpolitique était, de fait, plus en concordance avec sa théorie des contreforces et des points de résistance disséminés dans le corps social qu'avec une foi unitaire de gauche. Il essayait, avec d'évidentes difficultés, d'échapper à toute réduction de gauche et à tout verbalisme, de quelque bord qu'il futt, d'où ses efforts pour ouvrir de nouveaux champs d'intervention à l'action politique: comités d'action des prisonniers s'en prenant aux conditions effectives de détention, des homosexuels en quête de reconnaissance, mise en cause des hôpitaux psychiatriques, d'une certaine psychiatrie comme d'une certaine médecine... Tout cela se trouvant plus ou moins "récupéré"par un discours de gauche qui faisait alors flèche de tout bois mais se plaçant résolument en rupture avec les lourdeurs et les contraintes du discours marxiste dominant. Sur ces entrefaites, parut le livre de Jean Baudrillard qui était un nouvel essai de récupération, plus provoquant encore que les précédents en raison des prétentions de l'auteur.

\section{LE TRAVAILDE LA MORT}

(à propos de Jean Baudrillard: Oublier Foucault, Paris, Editions Galilée, 1977)

Jean Baudriilard, entamant une libre dérive à partir du dernier livre de Michel Foucault La volonté de savoir, prétend passer à la spire suivante de la spirale interprétative et nous contraindre ainsi à "oublier Foucault". Il reproche au discours propre à Michel Foucault de "produire" (c'est-à-dire à la fois de mettre en avant et de fabriquer) "une strophe sans origine" ni fin assignables, sans la moindre vacuité interstitielle et qui 
n'envisage en rien "letravail du négatif," "production" pure d'une analytique pleine et fluide du pouvoir--des pouvoirs--et qui emplit tout le champ social et historique considéré d'un émiettement actif de forces et de contreforces, pulvérisant à l'infiniles points d'articulation et de résistance. Mais Baudrillard est loin d'entendre "le travail du négatif" dans le sens hégélien d'une négation dialectique qu'il faudra à son tour nécessairement nier; il le conçoit comme séduction ou défi à mort, ${ }^{\text {is }}$ ce qui pour lui s'oppose à toute "production" de savoir-pouvoir. Au nom de la force toute-puissante de la séduction qu'il affirme, sans ambages, être le seul principe actif de subversion dans notre monde-miroir où se déploie la stratégie disséminatrice des simulacres et des reflets de reflets, il proclame doctement révolus (mais de par quel savoir-pouvoir?) la tentative et l'objet théoriques de Foucault parce que ce dernier prétendrait saisir à fleur d'archive des "effets de réel," des procédures de vérité, et, incrimine-t-il, ces effets comme ces procédures sont toujours, en dernier ressort, politiques. L'oeuvre entier de Michel Foucault ne serait que le mythe chatoyant d'un monde entièrement révolu, d'un monde dont il serait lui-même le simulacre.

Mais, pour disqualifier plus commodément l'entreprise de Foucault, il lui attribue un champ d'investigation qui n'est pas exactement le sien: il situe en effet les analyses de Foucault au niveau des seuls "effets de réel" et des procédures de vérité tels qu'ils se manifesteraient dans le tissu social et historique même, alors que Foucault a dessein--et il le répète avec constance depuis L'archéologie du savoir--de se situer à la frange mouvante où s'engrènent institutions et discours--discours eux-mêmes institutionnels ou discours sur les institutions. En ce qui regarde la sexualité, il annonce "une série d'études concernant les rapports historiques du pouvoir et du discours sur le sexe". Il souhaite mettre au jour ("produire" si l'on veut, au sens de révéler) une "technologie du sexe", c'est-à-dire montrer l'intrication particulière de discours (religieux, moraux, médicaux, sociaux...) et d'actes socialement codés qui prend dans notre histoire le nom de sexualité--il s'agit bien d'une "technologie" en effet et non d'une "technique": il y a là en plus le "logos",discours et logique du sexe. Le travail analytique de Foucault tente de démêler sans cesse l'écheveau des "effets de réel" et des effets de discours: il n'ignore pas, à

\footnotetext{
${ }^{13}$ Grand champion de l'anathème en tout genre, Baudrillard est litteralement fasciné par l'importance symbolique, pratique et physique de la mort dans notre monde moderne: l'idée de séduction qui est son maître mot est perte et déviance, dérapage semi-conscient vers l'abolition et l'effacement, la violente puissance d'attraction-répulsion propre à la mort et a la destruction--Thanatos--est sans doute pour lui la séduction-même.
} 
ce niveau, le jeu des simulacres, l'occultation fragmentaire du réel par le discours (et réciproquement). Par exemple, dans la quête éperdue de sexe et de jouissance qui démène notre époque, Foucault met clairement en évidence la stratégie de l'aveu, fondée sur le simulacre intériorisé du secret, et il fait éclater que le seul secret en la matière est qu'il n'y en a pas. De même il démonte pour nous les simulacres de la "répression" comme de la "libération" sexuelles.

Sur ces points précis d'ailleurs, Baudrillard n'attaque guère Foucault, il surenchérit même sur ses analyses--ne critiquant que ce qu'il estime rester flou dans le concept de micropouvoirs et reprenant avec lui, l'amplifiant, la dénonciation du pousse-au-jouir forcené et contraignant propre à nos jours, au profit d'une réelle "transversalité" du désir. Mais cette surenchère lui permet, pense-t-il, de laisser l'oeuvre de Foucault derrière lui comme le cénotaphe d'un savoir-pouvoir déjà éteint: il propose le travail de la séduction (tout ce qui détourne et cache au lieu d'expliquer et de produire). Il veut se situer par-delà la mort du pouvoir grâce au formidable pari d'un défi à mort: car la révolution a déja eu lieu," "elle s'est dejà recourbée sur elle-même pour produire son simulacre...." Baudrillard se veut déjà sur l'autre versant du cercle (ou du cycle) ainsi dessiné et il porte au pouvoir ce "défi à mort": "c'est le défi à ceux qui détiennent le pouvoir d'assumer ce pouvoir jusqu'à la limite, qui ne peut être que la mort des dominés"--et donc l'extinction du pouvoir.

Et Baudrillard ici retotalise, recentralise le pouvoir, nommant l'ennemi d'un nom unique, alors que Foucault déterritorialise, décentre, dissémine, expliquant comment le pouvoir est passé de la guerre du souverain contre ses sujets à la gestion de plus en plus paisible des potentialités propres au cheptel social. Baudrillard semble figé dans l'attente magique, "angéliste" peut-être, de l'écroulement du pouvoirsimulacre happé par la logique manichéenne du défi et s'autodétruisant. Ce n'est en fait qu'une manière de demeurer en-deçà de Foucault, un retour à des totalisations déjà-là bien que frénétiques, une stratégie de provocation et d'attente à laquelle la seule réponse semble être la mort: le défi évoqué et invoqué par Baudrillard n'est que le travail de la mort, mais d'une mort qui n'a aucunement l'efficace du négatif hégélien ni de l'excès propre à la pensée de Georges Bataille, une mort qui est pure perte et perte pure dans le silence redondant des simulacres quotidiens. La pensée de Michel Foucault n'est pas pour nous qu'une péripétie: nous ne sommes pas encore prêts à oublier Foucault!

(juin 1977) 
La réaction de Foucault à ce livre fut une réaction de mépris. Il évoqua "tous ces gens, incapables de travaillerpar eux-mêmes et de discuter sérieusement, [qui] ne cherchent d'existence que dans des polémiques, dénonciations, mises à mort" (lettre du 29 juillet 1977). Toutefois certains arguments du livre de Baudrillard avaient avivé d mes yeux l'importance d'une difficulté qui me paraissait alors cruciale: ne fallait-il pas envisager dans le champ social, tel que le concevait La volonté de savoir, une instance d' "arbitragequi permit aux multiples effets de pouvoirs et de contrepouvoirs en dissémination (ou en fusion) d'atteindre une cohésion d'ensemble suffisante pour éviter l'anarchie ou un éparpillement insensé? N'y avait-il pas un effet (ou des effets) de pouvoir "remontant" de la base (les micropouvoirs) vers un sommet (le Pouvoir, un faisceau de pouvoirs?) et qui pussent expliquer la hiérarchiedes forces et des formes sociales et politiques telle que nous la constatons? Il me semblait en effet que l'insistanceplacée sur la pulvérisation des pouvoirs et des points de résistance s'accordait mal avec l'analyse faite par ailleurs des grands mouvements ou des tendances dominantes qui caractérisent telle ou telle période, telle ou telle épistémè. Foucault m'écrivit ceci qui voulait répondre d̀ la fois à mon inquiétude et aux prétentions de Baudrillard: "Quant à la question de l'"arbitrageque vous posez d propos des effets de pouvoir 'remontant', je voudrais montrer qu'il s'agitplutôt de saturation stratégique (ce qui est tout autre chose)". J'avoue que l'expression, plutôt énigmatique, de "saturation stratéqique" me fait encore souvent rêver. Dans les années qui suivirent, la recherche de Foucault s'infléchit et prit l'allured'un plus vaste détour. Ce qui devait être l'histoire de la sexualité considérée dans les trois derniers siècles de nos temps modernes se métamorphosa en une quête des origines de la "chair" et nécessita un retour décisif d̀ l'antiquité: "Je me suis vite aperçu qu'on ne pouvait rien comprendre d̀ la conception chrétienne de la chair sans une analyse un peu sérieuse des textes patristiques. Ce que je fais. Ce que je suis en train d'achever. Ce qui sera fini cet été, juré, craché" (lettre du 20 février 1981). Ce ne fut, on le sait, pas tout à fait le cas.

Université de La Réunion

SERGE MEITINGER 